\title{
Consensus of the Characteristics of Clinical Judgement Utilised by Nurses' in Their Practice: Results of a Survey
}

\author{
Sharon Jacobs*, Christine Taylor, Kathleen Dixon, Lesley Wilkes \\ School of Nursing and Midwifery, Western Sydney University, Sydney, Australia \\ Email: *s.jacobs@westernsydney.edu.au
}

How to cite this paper: Jacobs, S., Taylor, C., Dixon, K. and Wilkes, L. (2018) Consensus of the Characteristics of Clinical Judgement Utilised by Nurses' in Their Practice: Results of a Survey. Open Journal of Nursing, 8, 746-757.

https://doi.org/10.4236/ojn.2018.810056

Received: September 19, 2018

Accepted: October 27, 2018

Published: October 30, 2018

Copyright ( 92018 by authors and Scientific Research Publishing Inc. This work is licensed under the Creative Commons Attribution International License (CC BY 4.0).

http://creativecommons.org/licenses/by/4.0/

\begin{abstract}
Clinical judgement is an important component of safe and effective nursing care. Clinical judgement remains a challenging cognitive process to articulate and assess. An integrative review of the literature conducted in 2016 identified 13 characteristics of clinical judgement. This paper reports the results of a 2-phase survey to obtain consensus whether these characteristics identified accurately represented nurses' perception of clinical judgement. The 2-phase survey was administered via an online survey software tool. The survey in phase 1 sought Yes/No answers for nurse academics with recruitment via a hyperlink in an email to individuals identified from University websites, Google and published articles. In phase 2, the survey was modified to a Likert scale and recruitment occurred via online networks and was open to all nurses. The results identified that the characteristics accurately represented the nurse's perception of clinical judgement. By articulating the characteristics, it allows clinical judgement to be easily described and can therefore be applied to both nursing research and education.
\end{abstract}

\section{Keywords}

Clinical Judgement, Characteristics, Survey, Nurses

\section{Introduction}

Clinical judgement has been identified as an essential component of safe and effective nursing care and has been incorporated into the competency standards for registration by various nursing registration boards [1] [2] [3] [4] [5]. As nurses engage in multiple clinical judgement episodes in their care of a patient, it is paramount that nurses have the ability to recognize important changes in the 
patient's condition, especially in the deteriorating patient, and respond appropriately [6] [7]. However, nurse leaders have identified that new graduate nurses may not always adequately demonstrate clinical judgement in practice [8] [9] [10] [11].

Often the definitions and characteristics of clinical judgement are not clearly stated in the literature [12]. Clearly identified characteristics could provide a framework for clinical judgement to assist both clinical nurses and nurse researchers in observing and describing clinical judgement in action.

This paper reports a study that aimed to clarify the main characteristics of clinical judgement from the perspectives of nurse scholars, nurse academics and clinical nurses.

\section{Background}

Although clinical judgement has been identified as an important component in the provision of safe and effective patient care, it remains a challenging concept to articulate and assess. Clinical judgement in nursing is broadly described as involving the collection of important patient data, the interpretation of the collected data, forming a hypothesis, responding by nursing action and reflecting on and evaluating the actions taken [7] [13] [14] [15].

The most commonly used definition of clinical judgement in the nursing literature is that of Tanner [7] who describes the cognitive processes nurses use to determine and anticipate patient's needs. This definition was developed from her observation of expert nurses and their decision-making processes. Furthermore Tanner [7] identified what the nurse brings to the situation (their knowledge and experience), how a relationship with a patient, (knowing the patient and how they respond) as well as the context in which the situation occurs, all of which will influence that nurse's clinical judgement. While this definition describes the concept of clinical judgement, the specific characteristics or elements that are incorporated within this process were not stated.

A search of the literature revealed two separate concept analyses that described the characteristics or specific elements of clinical judgement [12] [16]. A concept analysis is usually undertaken to identify the basic element or characteristic of a concept [17]. Delineating the concept of clinical judgement into its characteristics will allow the objective observation of clinical judgement in action to measure students' achievements in this skill and to identify areas for further development.

The concept analysis by South African researchers van Graan, Williams [16] aimed to develop a theoretical definition of clinical judgement using a framework by Walker and Avant. Following a search of the literature (including both medical and nursing dictionaries) the researchers defined clinical judgement as both a singular event "Clinical judgement is the conclusion at which a nurse arrives through the ability to gather salient pieces of information ..." (p. 38), and a process, with 18 characteristics (or attributes) of the concept of clinical judgement (p. 39). A second concept analysis conducted by Australian researchers 
[12] in an integrative review identified 13 characteristics.

The characteristics from both studies have at their core the collection and interpretation of data, planning of care, and evaluation of the data. This is consistent with Tanner's definition of clinical judgement described earlier. Both sets of characteristics also emphasised the theoretical, practical and experiential knowledge of the nurse and the context within which the clinical judgement occurs, which are important aspects of clinical judgement according to Tanner [7]. However, in the van Graan paper the characteristics are not mutually exclusive, for example, "manage uncertainty" is a synonym for the characteristic "nuanced/ distinct ability" and also as the separate characteristic "managing uncertainty". van Graan, Williams [16] described antecedents and consequences around the action of clinical judgement, so clinical judgement appears to be separate to the characteristics and therefore the nature of clinical judgement appears unclear.

Clearly there remains uncertainty around the characteristics of clinical judgement and more research is needed to enable educators and researchers to explore and apply the concept of clinical judgement. To further develop this field of research, the authors undertook a study to clarify the characteristics formulated from the concept analysis in our previous paper [12]. The current paper reports a two-phase survey to obtain consensus from nurses in both academic and clinical roles about the characteristics of clinical judgement.

\section{Aim}

The aim of the study was to:

- Confirm the characteristics of clinical judgement identified from the literature;

- Identify if the characteristics accurately represented the nurses' perceptions of clinical judgement.

\section{Method}

\subsection{Design}

The study used an online survey developed by the researchers to investigate whether the characteristics of clinical judgement accurately represented the perceptions of clinical judgement used by nurses in their clinical practice. There has been an increase in use of online surveys as the use of the internet has become widespread [18] [19]. The use of online surveys has the advantages of potentially being able to access populations in distant locations and can reduce the time and effort exerted by researchers [18].

The study used a survey design in two phases using convenience sampling with participants invited to complete the survey online. The survey instrument sought demographic information and identification as to whether the previously identified 13 characteristics [12] accurately represented the cognitive process of clinical judgement. 


\subsection{Ethics}

Prior to data collection approval from the institutional Human Research Ethics Committee was obtained. As data were collected using an anonymous online survey therefore confidentiality was assured. Implied consent was indicated by completion of the survey.

\subsection{Phase 1}

In phase 1 the survey incorporated a tick box format using Yes and No answers eliciting responses from nurse scholars and nurse academics. The survey during this phase was conducted between November 2015 to March 2016.

\subsection{Sample and Recruitment}

Participants in this phase of the study were nurses who work within the tertiary education sector. The participants were identified as nurse scholars: these were academics who had published around cognitive processes used by nurses and had a track record of research in the area with their information sourced from Google and published articles. This sample also included nurse educators identified from staff lists on university schools of nursing websites throughout Australia. Recruitment occurred through the inclusion of a hyperlink in an email.

\subsection{Survey Tool}

A survey was created asking participants whether they agree by answering either Yes/No to the 13 characteristics identified were components of clinical judgement. The 13 characteristics were: "essential for safe practice", "Interpretation of patient/clients' needs", "Culminates in a clinical decision", "Involves pattern recognition", "Knowledge based on practical skills", "Context Dependent", "Evaluation of choices made", "Knowledge based on experience", "Systematic process", "Reflective process", "Culminates in a clinical management plan", "Knowledge based on theory" and "cyclical process" [12].

\subsection{Data Collection}

Data were collected using an online survey software tool, Qualtrics ${ }^{\oplus}$ (http://www.qualtrics.com/research-suite/\#academic). The online survey is flexible and allows the collection of both quantitative and qualitative data and has the capacity for downloading the completed responses into suitable software for data analysis. The online survey was disseminated via individual emails to the nurse academics and accessible via an attached hyperlink. A total of 269 surveys were emailed with 39 completed surveys.

\subsection{Data Analysis}

Data analysis was performed on the survey responses of the 13 characteristics and a percentage agreement was calculated. There are no clear guidelines or objective methods available in the literature to identify the acceptable level of 
agreement between participants when completing surveys [20] [21]. When the survey is limited to only two scores (as this phase of the survey has Yes or No answers), percent agreement is a valid measurement to use [22].

\subsection{Results}

Demographics

Fifteen $(38 \%)$ of the respondents elected not to reveal their age; of the rest 41 50 years' group had the highest representation with 13 (33\%). Doctorate qualification was highly represented in the sample $(n=8)$ and the respondents held various positions with the university sector from associate lecturer to professor in nursing (Table 1).

\subsection{Survey}

As a Yes/No response was used it is difficult to determine what percentage of Yes determines a level of agreement as the literature varies from 50 upwards [23]

Table 1. Phase 1 Demographics of academics for combined nurse scholars and nurse academics.

\begin{tabular}{|c|c|c|}
\hline \multicolumn{2}{|c|}{ Characteristic } & \multirow{2}{*}{$\begin{array}{c}\text { Count and \% } \\
5(19 \%)\end{array}$} \\
\hline \multirow{5}{*}{$\begin{array}{c}\text { Age (years) } \\
\text { (valid } n=27 \text { ) }\end{array}$} & $21-30$ & \\
\hline & $31-40$ & $13(48 \%)$ \\
\hline & $41-50$ & $6(22 \%)$ \\
\hline & $51-60$ & $3(11 \%)$ \\
\hline & $>61$ & \\
\hline \multirow{4}{*}{$\begin{array}{l}\text { Highest Qualification } \\
\quad(\text { valid } n=22)\end{array}$} & Doctorate & $8(36 \%)$ \\
\hline & Master's & $7(32 \%)$ \\
\hline & Degree & $3(14 \%)$ \\
\hline & Diploma & $4(18 \%)$ \\
\hline \multirow{4}{*}{$\begin{array}{l}\text { Years in Nursing } \\
\text { (valid } n=16 \text { ) }\end{array}$} & $10-20$ & $6(38 \%)$ \\
\hline & $21-30$ & $6(38 \%)$ \\
\hline & $31-40$ & $3(19 \%)$ \\
\hline & $>40$ & $1(6 \%)$ \\
\hline \multirow{5}{*}{$\begin{array}{l}\text { Qualification } \\
\text { (valid } n=23 \text { ) }\end{array}$} & Certificate in Nursing & $1(4 \%)$ \\
\hline & Diploma in Nursing & $3(13 \%)$ \\
\hline & Degree in Nursing & $3(13 \%)$ \\
\hline & Master's degree & $7(30 \%)$ \\
\hline & Doctorate & $9(39 \%)$ \\
\hline \multirow{5}{*}{$\begin{array}{l}\text { Current position } \\
(\text { valid } n=23)\end{array}$} & Associate Lecturer A & $9(39 \%)$ \\
\hline & Lecturer B & $9(39 \%)$ \\
\hline & Lecturer C & $2(9 \%)$ \\
\hline & Associate Professor & $1(4 \%)$ \\
\hline & Professor & $2(9 \%)$ \\
\hline
\end{tabular}


[24], the authors elected to accept a consensus level of $60 \%$ (indicating a moderate level of agreement) as acceptable for each of the 13 characteristics in phase 1 of the survey (see Table 2). At this level of agreement all participants identified that 12 of the 13 characteristics were components of clinical judgement. Two of the 13 characteristics "interpretation of the patient's needs and "knowledge based on experience" had the highest level of agreement with $95 \%$. This was followed by "essential for safe patient/client care" at $92 \%$. There was a moderate level of agreement with five of the characteristics "knowledge based on theory, "evaluation of choices made, and actions taken", "reflective process", pattern recognition" having 67\% agreement and "systematic process" having 61\% agreement. The characteristic "a cyclical process" was only identified by $54 \%$ of respondents and fell below the level of agreement for acceptance as a perceived characteristic of clinical judgement.

\subsection{Phase 2}

In phase 2 the survey was adapted to use a 5-point Likert scale to indicate the extent of their agreement or disagreement with each of the characteristics identified. Participants in phase 2 were nurses from both clinical and academic areas. The survey for this phase was conducted between May 2017 and February 2018.

\subsection{Sample and Recruitment}

To identify the strength of agreement between respondents to the characteristics in the survey it was modified to indicate the extent of the agreement or disagreement with each of the 13 characteristics. The participants for this phase were all nurses with recruitment occurring via online networks with a hyperlink contained within the invitations posted to these networks.

Table 2. 60\% agreement for characteristics of clinical judgement in descending order.

\begin{tabular}{cc}
\hline Characteristics of clinical judgement & Count and \% agreement (N = 39) \\
\hline Interpretation of patient/client needs & $37(95 \%)$ \\
Knowledge based on experience & $37(95 \%)$ \\
Essential for safe patient/client care & $36(92 \%)$ \\
Culminates in a clinical decision & $32(82 \%)$ \\
Context dependent & $31(79 \%)$ \\
Knowledge based on practice skills & $30(77 \%)$ \\
Culminates in a nursing management plan & $29(74 \%)$ \\
Knowledge based on theory & $26(67 \%)$ \\
Reflective process & $26(67 \%)$ \\
Evaluation of choices made, and actions taken & $26(67 \%)$ \\
Systematic process & $26(67 \%)$ \\
& $24(61 \%)$
\end{tabular}




\subsection{Data Collection}

The software program Qualtrics ${ }^{\circledast}$ was used to collect the data in this phase. A5-point Likert scale was introduced with 1 being strongly disagree, 2 somewhat disagree, 3 neither agree or disagree, 4 somewhat agree, and 5 strongly agree with each of the 13 identified characteristics of clinical judgement. Likert scales have been widely used in research when assessing attitudes, as it permits a range of answers allowing the respondents to specify their level of agreement or disagreement with statements made [25].

The link to the survey was posted onto professional networks (Australian Nurse Teachers' Society \& Sigma Theta Tau International) and to social media (Facebook and Twitter), inviting all registered nurses to respond, and the survey remained open for two months. This captured nurses in a variety of sectors to confirm and validate the results of the first survey.

\subsection{Data Analysis}

The data were exported from Qualtrics ${ }^{\oplus}$ to Statistical Package for Social Sciences (SPSS) version 24 for management and analysis [26]. The demographics were presented as frequency distributions. Descriptive analysis of each characteristic was undertaken with frequencies tallied, means and standard deviation calculated. Cronbach's alpha was calculated for the items in the survey.

\subsection{Results}

\section{Demographics}

While seventy-six participants started the phase 2 survey and only completed the demographics section; 29 completed the full survey. From those that completed the full survey, the majority (72\%) were aged between $41-60$ years. Fourteen (48\%) respondents were in the 51 - 60 years' group with 7(24\%) in the $41-50$ years' group. The length of time the respondents worked in nursing ranged between 5 to 42 years with 15 (52\%) having between 30 - 45 years' nursing experience. Ten (34\%) respondents indicated a master's degree in nursing as their highest academic qualification. The positions these respondents held were varied, with nurse educators 10 (34\%) having the greatest representation (see Table 3). Twelve (41\%) respondents were currently employed in the hospital setting and eight (28\%) were community based. Eight (28\%) respondents identified which department they worked in which included intensive care (2), coronary care, operating theatre, emergency, endoscopy, recovery and justice/mental health. All the respondents except two spoke English as their main language.

\subsection{Survey}

The means, standard deviations and percentage of strongly agree/agree for each of the 13 items in the survey are presented in Table 4. Cronbach's Alpha for the overall consistency for the survey is 0.854 presented in Table 5 .

The 13 individual characteristics elicited "strongly agree" or "agree" responses from more than $79 \%$ of respondents with seven of the items eliciting a response 
Table 3. Demographics for Phase 2.

\begin{tabular}{|c|c|c|}
\hline \multicolumn{2}{|r|}{ Characteristic } & \multirow[t]{2}{*}{ Count and $\%(\mathrm{~N}=29)$} \\
\hline & mean & \\
\hline & range & \\
\hline & $21-30$ & $1(3 \%)$ \\
\hline \multirow[t]{6}{*}{ Age (years) } & $31-40$ & $5(17 \%)$ \\
\hline & $41-50$ & $7(24 \%)$ \\
\hline & $51-60$ & $14(48 \%)$ \\
\hline & $61-70$ & $2(7 \%)$ \\
\hline & Range & \\
\hline & $5-10$ & $3(10 \%)$ \\
\hline \multirow[t]{5}{*}{ Years in nursing } & $11-20$ & $2(5 \%)$ \\
\hline & $21-30$ & $12(41 \%)$ \\
\hline & $31-40$ & $8(26 \%)$ \\
\hline & $41-50$ & $3(10 \%)$ \\
\hline & Certificate in Nursing & $5(17 \%)$ \\
\hline \multirow{8}{*}{ Qualification } & Degree in nursing & $9(31 \%)$ \\
\hline & Master's Degree & $10(34 \%)$ \\
\hline & Doctorate & $4(14 \%)$ \\
\hline & Registered Nurse & $2(7 \%)$ \\
\hline & Registered Nurse/sessional academic & $1(3 \%)$ \\
\hline & Clinical Nurse Specialist & $1(3 \%)$ \\
\hline & Clinical facilitator & $2(5 \%)$ \\
\hline & Clinical Nurses Educator & $3(10 \%)$ \\
\hline \multirow[t]{7}{*}{ Current position } & Clinical Nurse Manager & $1(3 \%)$ \\
\hline & Nurse educator & $10(34 \%)$ \\
\hline & $\begin{array}{c}\text { Nurses Educator/sessional tutor } \\
\text { Lecturer }\end{array}$ & $1(3 \%)$ \\
\hline & Senior Lecturer & $4(14 \%)$ \\
\hline & Director of training/sessional academic & $1(3 \%)$ \\
\hline & Learning and development manager & $1(3 \%)$ \\
\hline & Hospital & $12(41 \%)$ \\
\hline \multirow[t]{2}{*}{ Current place of work } & Area Health Service & $8(26 \%)$ \\
\hline & University & $7(24 \%)$ \\
\hline
\end{tabular}

of $90 \%$ and above with the response for the characteristic "involves pattern recognition" was $97 \%$ and "knowledge based" was $96 \%$. The means and standard deviations for each of the characteristics range from a high of 4.79 ("essential for safe practice") to a low of 4.10 ("knowledge based on theory") (see Table 4). 
Table 4. Means, standard deviation and frequency scores for Phase 2 survey (in descending order).

\begin{tabular}{cccc}
\hline Characteristic & \multicolumn{2}{c}{$\begin{array}{c}\text { Means and Standard } \\
\text { deviations (SD) }\end{array}$} & $\begin{array}{c}\text { Frequency Strongly } \\
\text { agree or agree }\end{array}$ \\
\hline Mean & SD & $90 \%$ \\
Essential for safe practice & 4.79 & 0.55 & $93 \%$ \\
Interpretation of patient/clients' needs & 4.62 & 0.61 & $93 \%$ \\
Culminates in a clinical decision & 4.55 & 0.62 & $97 \%$ \\
Involves pattern recognition & 4.52 & 0.56 & $96 \%$ \\
Knowledge based on practical skills & 4.48 & 0.68 & $90 \%$ \\
Context Dependent & 4.41 & 0.67 & $86 \%$ \\
Evaluation of choices made & 4.31 & 0.79 & $93 \%$ \\
Knowledge based on experience & 4.28 & 0.58 & $79 \%$ \\
Systematic process & 4.28 & 0.87 & $86 \%$ \\
Reflective process & 4.28 & 0.78 & $82 \% \%$ \\
Culminates in a clinical management & 4.21 & 0.71 & $79 \%$ \\
plan & 4.10 & 0.71 &
\end{tabular}

Table 5. Cronbach's alpha reliability statistics.

\begin{tabular}{ccc}
\hline Cronbach's Alpha & Cronbach's Alpha Based on Standardized Items & N of Items \\
\hline 0.854 & 0.844 & 13 \\
\hline
\end{tabular}

\section{Discussion}

The aim of this study was to establish if the previously identified characteristics of clinical judgement by our team [12] accurately represented the perceptions of clinical judgement by nurse educators, academics and clinicians using an online survey. This aim was achieved as over $60 \%$ of the nurse academic and nurse scholar respondents in phase 1 identified 12 of the 13 characteristics as being characteristics of clinical judgement. The characteristic that had a low agreement was a "cyclical process". This is interesting as the respondents did identify that clinical judgement is a reflective process and that it involved evaluating the care given and making changes to the patient's management plan, which according to Nielsen, Stragnell [27] does infer a cyclical process.

This study's findings support previous literature where scholars suggest clinical judgement is essential for safe and effective nursing care [7] [15] [28] [29] [30]. While safe and effective care can be observed it can also be viewed as an outcome and thus needs further exploration. Interestingly, the characteristic with the highest percent agreement was "pattern recognition and synthesis" which is the ability for the early recognition of changes in the patient's condition which [28] identified as the difference between an expert and a novice nurse.

Including two surveys in the study design helped to identify the characteristics 
more definitively more than a single survey. Generally, there was variability in the responses between the two phases. In phase 1 the characteristics which scored the highest were "interpretation of patient/client's needs" and "knowledge based on experience" whereas in phase 2, "pattern recognition and synthesis" and "knowledge based on practical skills" scored the highest. Differences in responses could be reflective of the measure as the phase 1 survey forced a Yes/No response whereas in phase two respondents could report the degree to which they agreed with the characteristics. Also, there was a different context from which the participants viewed clinical judgement in phase 1 as the respondents were nurse academics and the second phase respondents were mostly clinicians (80\%). Benner, Tanner [28], Lasater [15] and Tanner [7] do state that clinical judgement is context dependent and therefore a variability of results would be expected. However, the study does show an agreement with the characteristics and reflects how important each characteristic is in relation to clinical judgement.

\section{Strengths and Limitations}

The strength of the study allows the characteristics of clinical judgement to be easily described so it can be applied to nursing research and education. Although there was the potential for larger numbers of participants using social and professional networks the response rate for the two phases of the survey remained low despite reminders. This is not uncommon when surveying nursing populations [31]. Despite a low response rate, the respondents were drawn from a variety of experienced nurses, both clinicians and nurse academics.

By using percentage agreement, while it is a simple and viable way to analyze the data as only two responses used, it does not provide a discrete analysis of participant agreement as a Likert scale.

\section{Conclusion}

The 13 characteristics identified accurately represented the nurses' perception of clinical judgement. Identifying the characteristics allows nurses and educators to describe the process of clinical judgement, might enable the identification of teaching strategies to promote the development of clinical judgement. To develop these strategies, all of the characteristics need to be incorporated.

\section{Recommendations}

The identification of the characteristics has increased the knowledge of the cognitive process of clinical judgement. This increase in the knowledge can be used to educate nursing students and guide the practice of registered nurses. When designing learning strategies and research projects to foster clinical judgement care needs to be taken to include all the identified characteristics.

\section{Conflicts of Interest}

The authors declare no conflicts of interest regarding the publication of this paper. 


\section{References}

[1] Manitoba (2012) Standards of Practice for Registered Nurses. College of Registered Nurses of Manitoba, Manitoba.

[2] Columbia, B. (2013) Professional Standards for Registered Nurses and Nurse Practitioners. College of Registered Nurses of British Columbia, Vancouver.

[3] NMC (2018) Future Nurses: Standards of Proficiency for Registered Nurses. Nursing and Midwifery Council, United Kingdom.

[4] NMBA (2016) Registered Nurses Standards for Practice. Nursing and Midwifery Board of Australia, Melbourne.

[5] ICN (2012) The ICN Code of Ethics for Nurses. International Council of Nurses, Geneva.

[6] Levett-Jones, T., et al. (2010) The "Five Rights" of Clinical Reasoning: An Edeucational Model to Enhance Nursing Students' Ability to Identify and Manage Clinically “at Risk” Patients. Nurse Education Today, 30, 515-520. https://doi.org/10.1016/j.nedt.2009.10.020

[7] Tanner, C. (2006) Thinking Like a Nurse: A Research-Based Model of Clinical Judgement in Nursing. Journal of Nursing Education, 45, 204-211.

[8] Kavanagh, J. and Szweda, C. (2017) A Crisis in Compentency: The Strategic and Ethical Imperative to Assessing New Graduate Nurses Clinical Reasoning. Nursing Education Perspectives, 38, 57-62. https://doi.org/10.1097/01.NEP.0000000000000112

[9] Monagle, J., et al. (2018) New Graduate Nurse Experiences in Clinical Judgment: What Academic and Practice Educators Need to Know. Nursing Education Perspectives. https://doi.org/10.1097/01.NEP.0000000000000336

[10] Missen, K., McKenna, L. and Beauchamp, A. (2016) Registered Nurses' Perceptions of New Nursing Graduates' Clinical Competence: A Systematic Integrative Review. Nursing and Health Sciences, 18, 143-153. https://doi.org/10.1111/nhs.12249

[11] El Haddad, M., Moxham, L. and Broadbent, M. (2013) Graduate Registered Nurse Practice Readiness in the Australian Context: An Issue Worthy of Discussion. Collegian, 20, 233-238. https://doi.org/10.1016/j.colegn.2012.09.003

[12] Jacobs, S., et al. (2016) Addressing the Challenge of Developing a Conceptual Definition for Clinical Judgment. Nursing and Health, 4, 1-8.

https://doi.org/10.13189/nh.2016.040101

[13] Phaneuf, M. (2008) Clinical Judgement-An Essential Tool in the Nursing Profession.

[14] Victor-Chmil, J. (2013) Critical Thinking versus Clinical Reasoning versus Clinical Judgement Differential Diagnosis. Nurse Educator, 38, 34-36. https://doi.org/10.1097/NNE.0b013e318276dfbe

[15] Lasater, K. (2011) Clinical Judgement: The Last Frontier for Evaluation. Nurse Edcuation in Practice, 11, 86-92. https://doi.org/10.1016/j.nepr.2010.11.013

[16] Van Graan, A., Williams, M. and Koen, M. (2016) Clinical Judgement within the South African Clinical Nursing Environment: A Concept Analysis. Health SA Gesondheid, 21, 33-45. https://doi.org/10.4102/hsag.v21i0.932

[17] Thompson, C. (2016) Nursing Theory and Philosophy Terms: A Guide. CJT Consulting \& Education, South Fork.

[18] Ahern, N. (2005) Using the Internet to Conduct Research. Nurse Researcher, 13, 55-69. 
[19] Wright, K. (2005) Researching Internet-Based Populations: Advantages and Disadvantages of Online Survey Research, Online Questionnaire Authoring Software Packages, and Web Survey Services. Journal of Computer-Mediated Communication, 10. https://doi.org/10.1111/j.1083-6101.2005.tb00259.x

[20] Cowman, S., et al. (2011) An International eDelphi Study Identifying the Research and Education Priorities in Wound Management and Tissue Repair. Journal of Clinical Nursing, 21, 344-353.

[21] Yaghmale, F. (2003) Content Validity and Its Estimation. Journal of Medical Education, 3, 25-27.

[22] McHugh, M. (2012) Interrater Reliability: The Kappa Statistic. Biochemia Medica, 22, 276-282. https://doi.org/10.11613/BM.2012.031

[23] Hasson, F., Keeney, S. and McKenna, H. (2000) Research Guidelines for the Delphi Survey Technique. Journal of Advanced Nursing, 32, 1008-1015.

[24] Xu, S. and Lorber, M. (2014) Interrater Agreement Statistics with Skewed Data: Evaluation of Alternatives to Cohen's Kappa. Journal of Consulting and Clinical Psychology, 82, 1219-1227. https://doi.org/10.1037/a0037489

[25] Gerrish, K. and Lacey, A. (2013) The Research Process in Nursing. John Wiley \& Sons, Somerset.

[26] Pallant, J. (2011) SPSS Survival Manual. 4th Edition, Allen \& Unwin, Crows Nest.

[27] Nielsen, A., Stragnell, S. and Jester, P. (2007) Guide for Reflection Using the Clinical Judgement Model. Journal of Nursing Education, 46, 513-516.

[28] Benner, P.R.N.P.F., Tanner, C.A.R.N.P.F. and Chesla, C.A.R.N.D. (2009) Expertise in Nursing Practice, Second Edition: Caring, Clinical Judgment, and Ethics. Springer Publishing Company, New York. https://doi.org/10.1891/9780826125453

[29] Fedko, A. (2016) Examining the Relationship between Clinical Judgement and Nursing Action in Baccalaureate Nursing Students, in School of Nursing. Indiana University, Ann Arbor, 176.

[30] Simmons, B. (2010) Clinical Reasoning: Concept Analysis. Journal of Advanced Nursing, 66, 1151-1158. https://doi.org/10.1111/j.1365-2648.2010.05262.x

[31] Luck, L., Ng Chok, H. and Wilkes, L. (2017) Nurses as Participants in Research: An Evaluation of Recruitment Techniques. Nurse Researcher, 25, 44-48.

https://doi.org/10.7748/nr.2017.e1546 